\title{
El cambio en las emociones de maestros en formación inicial hacia el clima de aula en una intervención basada en investigación escolar
}

\author{
Diego Armando Retana-Alvarado ${ }^{a}$, María Ángeles de las Heras Pérez $z^{\mathrm{b}}$, Bartolomé \\ Vázquez-Bernal ${ }^{\mathrm{c}}$, Roque Jiménez-Pérez ${ }^{\mathrm{d}}$. \\ Departamento de Didácticas Integradas. Universidad de Huelva. Huelva. España. \\ adiegoarmando.retana@alu.ubu.es,'bangeles.delasheras@ddcc.ubu.es,'bartolome.vazquez@ddcc.ubu.es \\ drjimenez@ddcc.ubu.es
}

[Recibido: 24 Noviembre 2017. Revisado: 18 Enero 2018. Aceptado: 19 Febrero 2018]

\begin{abstract}
Resumen: El clima de aula es un medio de interacción entre variables cognitivas, afectivas y sociales. Se describe el cambio en las emociones que experimentan futuros maestros hacia el clima de aula; antes, durante y después de la indagación de un problema sociocientífico sobre construcción hotelera en un Parque Natural de España. Participaron 54 estudiantes de Didáctica de las Ciencias de la Naturaleza I del Grado de Maestro en Educación Primaria de la Universidad de Huelva en el curso 2016-2017. Se aplicó un cuestionario de escala Likert en el que manifestaron su grado de acuerdo sobre la intensidad de catorce emociones. Los datos se sometieron a análisis descriptivo, análisis factorial exploratorio y pruebas no paramétricas. Las emociones positivas alcanzaron las intensidades más altas en las tres fases. Se establecen diferencias significativas en la mayoría de las emociones en función del momento. Se proponen implicaciones para la formación emocional de los maestros.
\end{abstract}

Palabras clave: Emociones; Clima de aula; Indagación; Conocimiento Didáctico del Contenido; Formación inicial de maestros.

The change in the emotions of teachers in initial formation towards the classroom climate in an intervention based on inquiry

\begin{abstract}
The classroom climate is a means of interaction between cognitive, affective and social variables. The purpose is to describe the change in the emotions experienced by teachers in initial training towards the classroom climate; before, during and after an inquiry of a socio-scientific problem about hotel construction in a Natural Park of Spain. Participated 54 students of the subject of Didactics of Nature Sciences I of the Degree in Primary Education of the University of Huelva in the academic year 2016-2017. A questionnaire of the Likert scale was applied in which the participants expressed their degree of agreement on the intensity of fourteen emotions. Data were subjected to descriptive analysis, exploratory factor analysis and non-parametric tests. Positive emotions reached the highest intensities in the three phases. Significant statistical differences are established in most of the positive and negative emotions depending on the moment. Implications are proposed for the emotional training of primary school teachers.
\end{abstract}

Keywords: Emotions; Classroom climate; Inquiry; Pedagogical Content Knowledge; Initial teachers training.

Para citar este artículo: Retana-Alvarado D. A., de las Heras Pérez M. Á., Vázquez-Bernal B., Jiménez-Pérez R. (2018) El cambio en las emociones de maestros en formación inicial hacia el clima de aula en una intervención basada en investigación escolar. Revista Eureka sobre Enseñanza y Divulgación de las Ciencias 15(2), 2602. doi: 10.25267/Rev_Eureka_ensen_divulg_cienc.2018.v15.i2.2602

\section{Introducción}

Gess-Newsome (2015) presenta un modelo constituido por conocimiento profesional del profesor y sobre tópicos científicos, amplificados o filtrados por concepciones, afectos y contexto que influyen en la enseñanza. El Conocimiento Didáctico del Contenido (CDC) se define como conocimiento base para planificación sobre un tópico y como habilidad. Shulman (1986) propone la interacción entre conocimiento de materia, pedagogía y currículum. En la enseñanza se establece un clima de aula; relaciones socioafectivas entre profesor y estudiantes

\author{
Revista Eureka sobre Enseñanza y Divulgación de las Ciencias \\ Universidad de Cádir. APAC-Eureka. ISSN: 1697-011X \\ bttp:/ / dx.doi.org/10.25267/Rev_Eureka_ensen_divulg_cienc.2018.v15.i2.2602 \\ http:/ / reuredc.uca.es
}


que implican creencias, emociones y comportamientos que permean y/o amplifican el aprendizaje.

Partimos de la Hipótesis de la Complejidad (Vázquez-Bernal et al. 2012), el profesor moviliza emociones a través de su modelo didáctico y CDC que influyen en reflexión, práctica y clima de aula; donde cambian las emociones de los futuros maestros en el transcurso de la intervención y en el aprendizaje sobre enseñanza de ciencias, particularmente sobre ecosistema. El conocimiento académico se integra y transforma en el CDC durante la enseñanza en el contexto particular (Mellado et al. 2014) y los cambios dependen de las emociones que experimentan los docentes durante la enseñanza de los contenidos científicos (Melo, Cañada y Mellado 2017).

La investigación como modelo didáctico alternativo (Cañal, Pozuelos y Travé 2005), facilita cómo pensar científicamente mediante formulación de preguntas, diseño de investigaciones, interpretación de datos y comunicación de argumentos. Este ámbito curricular permea todo el esquema del modelo, sienta sus bases en el conocimiento profesional de los tópicos y en la reflexión sobre y en la práctica. Las emociones inmersas en el CDC de quien aplica la indagación fundamentan el tópico e interacciona con el contexto.

En España, la investigación sobre dominio afectivo ha cubierto niveles básicos, formación inicial y desarrollo profesional (Brígido et al. 2013; Dávila et al. 2015; Retana-Alvarado et al. 2017). En el contexto internacional, se centra en regulación emocional de estudiantes en ciencias (Tomas, Rigano y Ritchie 2016), clima emocional en formación inicial (Bellocchi et al. 2014), emociones de maestros en tareas de investigación (Ritchie et al. 2013) y emociones en CDC de profesores en ejercicio (Melo et al. 2017). Los estudios se han diversificado en identidad profesional, inteligencia emocional e impacto sobre las emociones de los estudiantes (Uitto, Jokikoko y Estola 2015).

El objetivo es describir el cambio en intensidad de emociones que experimentan maestros en formación inicial frente al clima de aula; antes, durante y después de una intervención de un problema sociocientífico sobre el proyecto de construcción de hoteles en el interior de una zona protegida con alto valor ecológico.

\section{Marco Teórico}

\section{Las emociones en la enseñanza}

Los avances en neurociencia reconocen que una buena educación produce cambios en el cerebro que contribuyen en aprendizaje, emociones y desarrollo evolutivo (Mora 2016). Ante diversas circunstancias surgen reacciones biológicas y cognitivas que generan emociones; estas determinan decisiones, comportamientos y relaciones sociales; están constituidas por sentimientos, estimulación corporal, intenciones y aspectos comunicativos.

Fernández-Abascal (2009) las clasifica según valencia afectiva en emociones negativas y emociones positivas. Las primeras, se consideran desagradables, se manifiestan cuando se bloquea una meta y se caracterizan por tendencia a evitación; las segundas, son emociones agradables que se experimentan al alcanzar un objetivo y presentan tendencia de aproximación. Varían en cómo se experimentan, las personas intentan modificar cómo se sienten y ese proceso cognitivo es una estrategia de regulación emocional (Sutton y Harper 2009).

La enseñanza es una profesión emocional pues se establecen relaciones entre profesores y estudiantes. Las emociones influyen en toma de decisiones y desarrollo profesional, afecta sentimientos y acciones de otros con quienes interactúan, envuelve comprensión y labor 
emocional (Shapiro 2010). Afectan su comportamiento en estimulación cognitiva y motivacional, manejo del aula y soporte social. Su componente expresivo no pasa inadvertido por los estudiantes, están enlazadas al clima de aula, incluyendo comportamientos de estudiante y profesor (Frenzel 2014).

\section{Las emociones en la formación inicial y su relación con el clima de aula}

El clima de aula consiste en interacción de variables educativas, psicológicas, sociales, cognitivas, organizacionales y físicas que influyen en cómo los estudiantes aprenden (Alderman 2011), incide en valor predictivo de competencia emocional respecto al rendimiento académico (López-González y Oriol 2016) y tiene efectos positivos en autoeficacia y logro. Posee tres dimensiones: académica, relacionada con elementos pedagógicos y curriculares del entorno; gestión, referida a estilos disciplinares para mantener orden; y emocional, que comprende interacciones afectivas (Evans et al. 2009).

Para Bellocchi et al. (2014) es una experiencia de excitación afectiva entre grupos donde se conectan emociones y establecen interacciones para alcanzar aprendizaje. Otras dimensiones son aspectos físicos, orden y organización, contenido de lecciones y relaciones interpersonales. Hagenauer, Hascher y Volet (2015) indican que la capacidad de los maestros para conectar con los estudiantes se considera una habilidad para orientar la formación inicial, pues los maestros principiantes experimentan inseguridad. Ritchie et al. (2013) indican que en sus primeros años de enseñanza son vulnerables cuando experimentan emociones negativas debido a su dificultad para implementar la indagación.

Sus emociones hacia el aprendizaje de las asignaturas científicas influyen en las que vaticinan hacia la enseñanza, producto del recuerdo de sus antecedentes escolares. La mayoría recuerda curiosidad e interés hacia el aprendizaje de Biología y Geología; nerviosismo, tensión y preocupación hacia Física y Química. Vaticinan satisfacción y entusiasmo ante la docencia de Biología y Geología mientras que esperan sentir tensión, nerviosismo y preocupación ante Física y Química. Las emociones que vaticinan están correlacionadas con sus propias creencias de autoeficacia. Según el género, los varones experimentan emociones positivas con mayor frecuencia en aprendizaje y futura enseñanza de contenidos científicos. En función de la especialidad cursada en bachillerato, quienes estudiaron Ciencias, Tecnología o Ciencias de la Salud experimentan y vaticinan emociones más positivas a diferencia de aquellos que siguieron Humanidades, Ciencias Sociales o Arte (Brígido et al. 2013).

\section{Metodología}

\section{Tipo de estudio, problema e hipótesis}

La presente investigación longitudinal con alcance descriptivo y correlacional pretende responder al problema:

- ¿Cómo cambia la intensidad de las emociones positivas y negativas que experimentan los maestros en formación inicial respecto al clima de aula, antes, durante y después de una experiencia basada en investigación escolar?

A partir del problema se formulan las siguientes hipótesis:

- En el transcurso de toda la intervención, los maestros en formación inicial experimentan emociones positivas con mayor intensidad que las emociones negativas.

- Existen diferencias significativas en la intensidad de las emociones positivas y negativas en función del momento de la intervención. 


\section{Contexto}

El estudio nace y se desarrolla en la práctica de la docencia universitaria con estudiantes del Grado de Maestro en Educación Primaria, a quienes llamamos maestros en formación inicial. La presente investigación-acción se llevó a cabo a través del diseño y aplicación de una intervención basada en investigación de un problema sociocientífico sobre construcción de hoteles en terrenos protegidos de alto valor ecológico, incluido el Paraje Natural Enebrales de Punta Umbría (Huelva, España). Se analizaron implicaciones ambientales, sociales, económicas y políticas para aportar una posición argumentada. La intervención pretendía el aprendizaje de contenidos científicos y didácticos sobre ecosistema e investigación respectivamente, mediante un problema relevante y mal estructurado que requiere razonamiento científico basado en pruebas para informar decisiones y empleo de tópicos científicos con ramificaciones sociales para que se impliquen en diálogo, debate y argumentación (Zeidler 2014). Se buscaba despertar emociones y generar un clima emocional adecuado mediante estrategias de autorregulación como metáforas emocionales, meditación y reflexión.

\section{El problema sociocientífico}

En 2000 el Ayuntamiento de Punta Umbría entregó el proyecto de construcción de hoteles, campo de golf y residencias a una empresa turística. Eso generó polémica entre sectores sociales pues los terrenos protegidos forman parte de una zona no urbanizable debido a sus características ecológicas, paisajísticas y recreativas. El espacio es un ecosistema dunar costero que posee cuatro frentes diferenciados: dunas primarias y secundarias, bosque mixto de enebros y sabinas y de pinos piñoneros. Se ubican especies de flora y fauna catalogadas en peligro de extinción como enebro marítimo, tomillo carnoso y camaleón.

En el diseño e implementación de la intervención se consideraron las fases de investigación escolar que proponen Cañal et al. (2005):

- Fase de iniciación: Se recogieron concepciones previas sobre ecosistema costero y se estudiaron contenidos científicos (concepto de sistema, clasificación de seres vivos, ecosistema, componentes bióticos y abióticos, relaciones tróficas) y conocimientos didácticos acerca de naturaleza de ciencia e indagación. Se trabajaron fuera del aula mediante técnica colaborativa y de expresión emocional denominada La telaraña. Los maestros en formación se posicionan formando un círculo, la profesora sujeta un ovillo de lana mientras plantea una pregunta y lo lanza a un estudiante quien debe responder. Luego, este lo lanza a otro quien responde la siguiente. El proceso se repite hasta que todos quedan enlazados en una red. El último en quedarse con el ovillo expresa las emociones que siente y lo regresa a quien se lo entregó. El proceso se repite hasta que retorna a quien anteriormente lo lanzó.

Antes de la intervención se aplicó el cuestionario para diagnóstico de emociones y cuestionario abierto que indagaba sobre concepciones previas en torno al ecosistema costero. Los datos permitieron establecer como resultado una hipótesis de progresión que distingue niveles de partida, intermedios y de referencia. Se aportarán evidencias al respecto en futuras publicaciones.

- Fase de planificación: Incluye presentación y delimitación del problema sociocientífico. Observaron un vídeo titulado Paraje Natural Enebrales de Punta Umbría y leyeron una noticia. Seguidamente, formularon la pregunta investigativa y elaboraron un plan de trabajo grupal.

- Fase de búsqueda de información: Antes de la gira, buscaron información sobre Paraje Natural Enebrales de Punta Umbría. Se realizó una visita guiada, donde la profesora explicó características del ecosistema dunar costero, especies emblemáticas de flora y fauna en cada 
frente e influencia de componentes bióticos y abióticos. Recogieron y sistematizaron evidencias. Al llegar a la playa se realizó meditación para facilitar gestión emocional y se aplicó el instrumento de emociones. Se realizó un sorteo en el que a cada equipo le correspondió asumir un rol (vecinos, políticos, ecologistas, empresarios, maestros y pescadores) con posición a favor o en contra de la construcción urbanística.

- Fase de estructuración: En el aula estructuraron los datos y formularon argumentos a partir de evidencias y contraste con explicaciones alternativas. Elaboraron un póster científico para comunicar sus argumentos. A nivel grupal debatieron sus explicaciones desde los roles y posiciones. Finalmente, la profesora intercambió la posición a favor o en contra del proyecto por lo que cada grupo debió aportar un nuevo argumento promoviendo empatía.

- Fase de evaluación: Reflexionaron y establecieron conclusiones sobre la cuestión controverti$\mathrm{da}$, logros y dificultades de la metodología indagatoria. Respondieron cuestionarios sobre emociones después de la intervención.

\section{Muestra}

Se realizó un muestreo no probabilístico por accesibilidad y conveniencia. La muestra estuvo conformada por 54 maestros en formación inicial (grupo T1), procedentes de España (Huelva, Sevilla y Cádiz) incluidas tres estudiantes Erasmus de Italia y Alemania. El grupo T1 intervino en prueba piloto y validación de la versión final del instrumento; y en el estudio longitudinal sobre cambio de emociones considerando los momentos antes, durante y después. Respecto al sexo, 33\% son varones y $67 \%$ son mujeres (efecto de la titulación). Las edades oscilan entre 19 y 39 años, situándose la media en 22,16 (DE=4,10).

Estos sujetos cursaron la asignatura Didáctica de las Ciencias de la Naturaleza I (CCNN), correspondiente al tercer curso del Grado de Maestro en Educación Primaria de la Universidad de Huelva, en el primer cuatrimestre del año académico 2016-2017. La asignatura donde se desarrolló el estudio está adaptada al Espacio Europeo de Educación Superior, constituida por 6 créditos. Se desarrollaron cuatro unidades didácticas: a) ¿Por qué y para qué enseñar CCNN? b) ¿Qué enseñar en CCNN? c) ¿Cómo enseñar CCNN? d) ¿Cómo evaluar en CCNN?

La asignatura fue impartida por tres profesores (biólogos con formación en Didáctica de las Ciencias Experimentales y Doctorado en Botánica y Bioquímica) de forma independiente a cuatro grupos (T1, T2, T3 y T5) que en total representan 185 maestros en formación, quienes también intervinieron en la validación de la versión definitiva del instrumento. Los grupos T2, T3 y T5 participaron únicamente en esa última fase de construcción. Se utilizan estos códigos para identificar el turno asignado a cada grupo en ese curso.

\section{Instrumento}

Se utilizó un cuestionario diseñado y validado por los autores, que recoge las variables sociodemográficas (sexo, edad y procedencia) y el grado de acuerdo declarado por los maestros en formación inicial acerca de la intensidad de las emociones positivas y negativas que experimentan frente a seis dimensiones: asignatura Didáctica de las Ciencias de la Naturaleza I, contenidos científicos, metodología indagatoria, profesor, clima de aula y evaluación.

Este trabajo se centra en el cambio de las emociones respecto al clima de aula (ver anexo 1), considerando tres momentos: antes, durante y después. Para cada dimensión se formuló un ítem en el que los participantes deben manifestar su nivel de acuerdo frente a catorce variables (ver tabla 1) en una escala tipo Likert constituida por cinco valores: 1 (totalmente en desacuerdo), 2 (de acuerdo), 3 (ni de acuerdo ni en desacuerdo), 4 (de acuerdo) y 5 (totalmente 
de acuerdo). Indican el número que mejor describe intensidad de la emoción que sienten frente a cada dimensión. La inclusión de cinco valores en la escala busca incrementar confiabilidad y ajustar los datos a una distribución normal. En diversos estudios se ha confirmado que la confiabilidad aumenta cuando se utilizan de cinco a siete alternativas en la escala Likert (Matas, 2018).

En los tres momentos, la prueba inferencial no paramétrica de Kolmogorov Smirnov demostró que, para cada variable, las significancias son menores que el nivel de significación estadística del 5,00\% ( $\mathrm{p}$-valor $<, 05$ ); en consecuencia, se rechazan las hipótesis nulas $\left(\mathrm{H}_{0}\right)$ en todas las variables y se aceptan las hipótesis alternativas $\left(\mathrm{H}_{1}\right)$. Se establece que la muestra proviene de una población con distribución no normal.

Tabla 1. Clasificación de las emociones.

\begin{tabular}{cc}
\hline Emociones positivas & Emociones negativas \\
\hline Entusiasmo & Susto \\
Satisfacción & Temor \\
Interés & Enojo \\
Aceptación & Tensión \\
Tranquilidad & Frustración \\
Bienestar & Aburrimiento \\
Asombro & Rechazo \\
\hline
\end{tabular}

Las variables responden a la selección de aquellas emociones discretas con carácter académico que desempeñan un rol relevante en la enseñanza (Mellado et al. 2014). El instrumento incluye un glosario con la definición de las emociones para transmitir su significado a los sujetos de estudio, homogeneizar la comprensión de los afectos y alcanzar validez de contenido.

\section{Validación del cuestionario}

$\mathrm{El}$ instrumento fue validado a través del criterio experto de tres investigadores, quienes valoraron los ítems con respecto al constructo, brindaron recomendaciones sobre redacción e inclusión o descarte de algunas variables para alcanzar claridad, pertinencia y validez de contenido.

Se llevó a cabo prueba piloto con 53 maestros en formación inicial (grupo T1) al comienzo del curso. En ese momento, el ítem estaba formulado en función de las emociones que esperaban sentir. Las propiedades psicométricas fueron satisfactorias para emociones positivas y negativas, siendo emociones positivas las que alcanzaron mayor intensidad. En cuanto a fiabilidad interna del cuestionario, los valores alfa de Cronbach para emociones positivas $(\alpha=, 79)$ y emociones negativas $(\alpha=, 87)$ fueron suficientes y satisfactorios.

También, el instrumento se validó utilizando una muestra de 185 maestros en formación inicial que conformaban los cuatros grupos (T1, T2, T3 y T5) de la asignatura. Se registraron propiedades psicométricas óptimas para emociones positivas $(\alpha=, 81)$ y negativas $(\alpha=, 88)$. En esta validación se incrementó confiabilidad y precisión de la escala, reduciéndose el error de medida. Los datos se sometieron a análisis factorial exploratorio cuyo objetivo fue determinar la validez de constructo, basada en la estructura interna, en la dimensión clima de aula. Así, se establece la cantidad de factores que subyacen a los ítems y las saturaciones correspondientes a cada uno.

Partiendo de la totalidad de las puntuaciones de la muestra, se calculó matriz de correlaciones entre variables y se halló existencia de multicolinealidad (det=,001). Se observaron 76 correlaciones bivariadas, considerando el coeficiente tho de Spearman en niveles de significación $\mathrm{p}=, 001$ y $\mathrm{p}=, 05$. Los resultados en las pruebas de adecuación muestral Kaiser Meyer Olkin $(\mathrm{KMO}=, 887)$ y esfericidad de Barlett $\left(\mathrm{X}^{2}=1193,96\right)$ son significativos $(\mathrm{p}=, 000)$, 
por tanto brindan oportunidad para realizar dicha factorización.

La extracción de los factores se realizó empleando el método por componentes principales; todas las variables presentaron suficientes niveles de comunalidad. La factorización fue capaz de explicar más del 50,0\% de variabilidad en cada variable. Respecto al número de factores a extraer se consideraron tres criterios: raíz latente, porcentaje acumulado de varianza explicada próximo al $60,0 \%$ y contraste de sedimentación. Se rotaron los factores a través del método Varimax con normalización Kaiser y se interpretó una estructura factorial constituida por tres factores. El primero, con varianza explicada del 39,5\% se identifica como temor, constituido por correlaciones entre frustración, enojo, rechazo, susto, tensión y aburrimiento. El segundo factor es explicado por $17,4 \%$ y se denomina bienestar, acompañado de satisfacción, interés, entusiasmo, tranquilidad y aceptación, con las que correlaciona positivamente. El 7,00\% de la varianza explica el tercer factor identificado como asombro. En conjunto, representan $63,9 \%$ de la varianza total, valor aceptado en investigación exploratoria en Ciencias Sociales.

Tomando en cuenta la relación entre potencia estadística y tamaño muestral mayor a 150 sujetos, los tres factores presentan cargas factoriales $\geq$,45. A partir de puntuaciones factoriales de la matriz de componentes rotados, se obtuvo valores óptimos de Varianza Media Extraída $(\mathrm{VME}=, 582)$ y Fiabilidad Compuesta $(\mathrm{FC}=, 95)$, ambos por encima de los valores recomendados $(\mathrm{VME}=, 50$ y $\mathrm{FC}=, 70)$. Los resultados derivados de estas validaciones ofrecen respaldo metodológico y rigor estadístico suficiente que permiten justificar la aplicación del cuestionario en los momentos antes, durante y después.

\section{Procedimiento de recolección de datos}

La aplicación del cuestionario se llevó a cabo en octubre y diciembre de 2016 y enero de 2017, en correspondencia con los momentos antes, durante y después de la intervención. Todos los sujetos aceptaron participar y fueron informados acerca del objetivo, anonimato y confidencialidad.

\section{Técnicas de análisis de datos}

Para el vaciado y análisis se recurrió al SPSS, versión 23,0. Se realizó análisis descriptivo exploratorio para evaluar propiedades psicométricas (distribución, variabilidad, dispersión y fiabilidad) y frecuencias en cada variable. Se aplicó análisis factorial exploratorio para determinar validez de los instrumentos y confiabilidad de los factores. Dado que los datos carecen de normalidad ( $\mathrm{p}$-valor $=, 000$ ) se aplicó la prueba inferencial no paramétrica $\mathrm{H}$ de Kruskal Wallis para tres muestras no relacionadas al 95,0\% de confianza $(\mathrm{N}=162)$, para determinar si existen diferencias significativas entre antes, durante y después, a partir del cálculo de errores tipo I $(\alpha)$.

Con el objeto de identificar los pares de momentos en los que existen tales diferencias, se aplicó la prueba no paramétrica U de Mann-Whitney para dos muestras no relacionadas $(\mathrm{N}=108)$ en todas las variables. Finalmente, se estimó error tipo II $(\beta)$, potencia estadística mediante prueba de una cola y tamaño del efecto sobre diferencias significativas y no significativas para evaluar la intervención. El efecto sobre la U de Mann-Whitney se obtuvo a partir del cociente de la división entre el valor $\mathrm{U}$ y el producto del tamaño de ambas muestras. El cálculo del tamaño del efecto sobre la $\mathrm{H}$ de Kruskal Wallis se realizó mediante el coeficiente épsilon al cuadrado $\left(\mathrm{E}_{\mathrm{R}}^{2}\right)$.

\section{Resultados}

\section{Análisis descriptivo intragrupo}

Los maestros en formación inicial expresaron emociones positivas y negativas con diferentes 
intensidades que cambiaron a lo largo de la intervención (ver tabla 2 y figura 1).

Tabla 2. Cambio de las emociones de los maestros en formación inicial respecto al clima de aula

\begin{tabular}{ccccccccccccc}
\hline & \multicolumn{4}{c}{ Antes } & \multicolumn{4}{c}{ Durante } & \multicolumn{6}{c}{ Después } \\
\cline { 2 - 12 } & $M$ & $D E$ & $M d n$ & $S^{2}$ & $M$ & $D E$ & $M d n$ & $S^{2}$ & $M$ & $D E$ & $M d n$ & $S^{2}$ \\
\hline Susto & 1,54 &, 86 & 1,00 &, 74 & 1,15 &, 49 & 1,00 &, 24 & 1,24 &, 58 & 1,00 &, 34 \\
Temor & 1,56 &, 97 & 1,00 &, 93 & 1,15 &, 36 & 1,00 &, 13 & 1,35 &, 71 & 1,00 &, 49 \\
Enojo & 1,52 &, 86 & 1,00 &, 75 & 1,20 &, 56 & 1,00 &, 32 & 1,19 &, 48 & 1,00 &, 23 \\
Tensión & 1,83 &, 97 & 2,00 &, 93 & 1,46 &, 88 & 1,00 &, 78 & 1,37 &, 76 & 1,00 &, 57 \\
Frustración & 1,61 &, 92 & 1,00 &, 85 & 1,19 &, 44 & 1,00 &, 19 & 1,30 &, 57 & 1,00 &, 33 \\
Aburrimiento & 2,04 & 1,08 & 2,00 & 1,17 & 1,43 &, 69 & 1,00 &, 47 & 1,41 &, 79 & 1,00 &, 62 \\
Rechazo & 1,59 &, 79 & 1,00 &, 62 & 1,15 &, 60 & 1,00 &, 36 & 1,33 &, 64 & 1,00 &, 42 \\
Entusiasmo & 3,50 &, 99 & 4,00 &, 97 & 4,00 &, 73 & 4,00 &, 53 & 3,87 &, 91 & 4,00 &, 83 \\
Satisfacción & 3,54 &, 99 & 4,00 &, 97 & 4,11 &, 74 & 4,00 &, 55 & 4,06 &, 81 & 4,00 &, 66 \\
Interés & 3,74 &, 81 & 4,00 &, 65 & 4,06 &, 74 & 4,00 &, 54 & 4,06 &, 76 & 4,00 &, 58 \\
Aceptación & 3,74 &, 92 & 4,00 &, 84 & 4,13 &, 70 & 4,00 &, 49 & 4,15 &, 83 & 4,00 &, 70 \\
Tranquilidad & 3,59 &, 92 & 4,00 &, 85 & 4,04 &, 87 & 4,00 &, 75 & 4,09 &, 85 & 4,00 &, 73 \\
Bienestar & 3,56 & 1,02 & 4,00 & 1,04 & 4,06 &, 83 & 4,00 &, 69 & 4,06 &, 88 & 4,00 &, 77 \\
Asombro & 2,69 & 1,09 & 3,00 & 1,20 & 3,19 & 1,43 & 3,00 & 2,04 & 3,30 & 1,36 & 3,00 & 1,84 \\
\hline
\end{tabular}

En cuanto a las emociones negativas, los resultados muestran que susto, temor, enojo, tensión, frustración, aburrimiento y rechazo presentaron las intensidades más fuertes antes de la intervención, en contraste con las siguientes fases. Aburrimiento y tensión eran las emociones negativas con intensidades más elevadas. Seguidamente, disminuyó la intensidad de todas las emociones negativas cuando aburrimiento y frustración asumieron las diferencias más notorias respecto a la fase preliminar, lo cual es provechoso porque se persigue que las emociones negativas disminuyan. En ese instante, tensión reportó mayor intensidad en comparación con las demás emociones negativas.

Se evidencia que casi todas las emociones negativas aumentaron después de la mediación a diferencia con el momento intermedio. El mayor incremento se obtuvo en temor y la disminución más apreciable se registró en tensión. Después de la indagación, las emociones de mayor intensidad son aburrimiento y tensión. Finalmente, entre antes y después todas las emociones negativas disminuyeron, especialmente aburrimiento y tensión.

Las emociones positivas registraron medias más altas frente a emociones negativas a lo largo de toda la intervención. En el momento inicial se aprecia que aceptación e interés presentaron las más elevadas. En el momento intermedio, todas las emociones positivas aumentaron, especialmente satisfacción que experimentó el cambio más notorio y está acompañada de aceptación con la media mayor del conjunto positivo.

Después de la mediación, se aprecia que aceptación, tranquilidad y asombro incrementaron en relación con el momento durante, mientras que entusiasmo y satisfacción experimentaron una leve caída. Interés y bienestar no contribuyen al establecimiento de diferencias entre los momentos durante y después, es decir, mantienen intensidades constantes. Las diferencias entre ambos momentos son apenas apreciables.

Existe un cambio favorable de todas las emociones positivas dado que incrementaron entre los momentos antes y después. Las diferencias entre ambos son más evidentes en asombro, satisfacción, tranquilidad y bienestar a favor del momento final. 


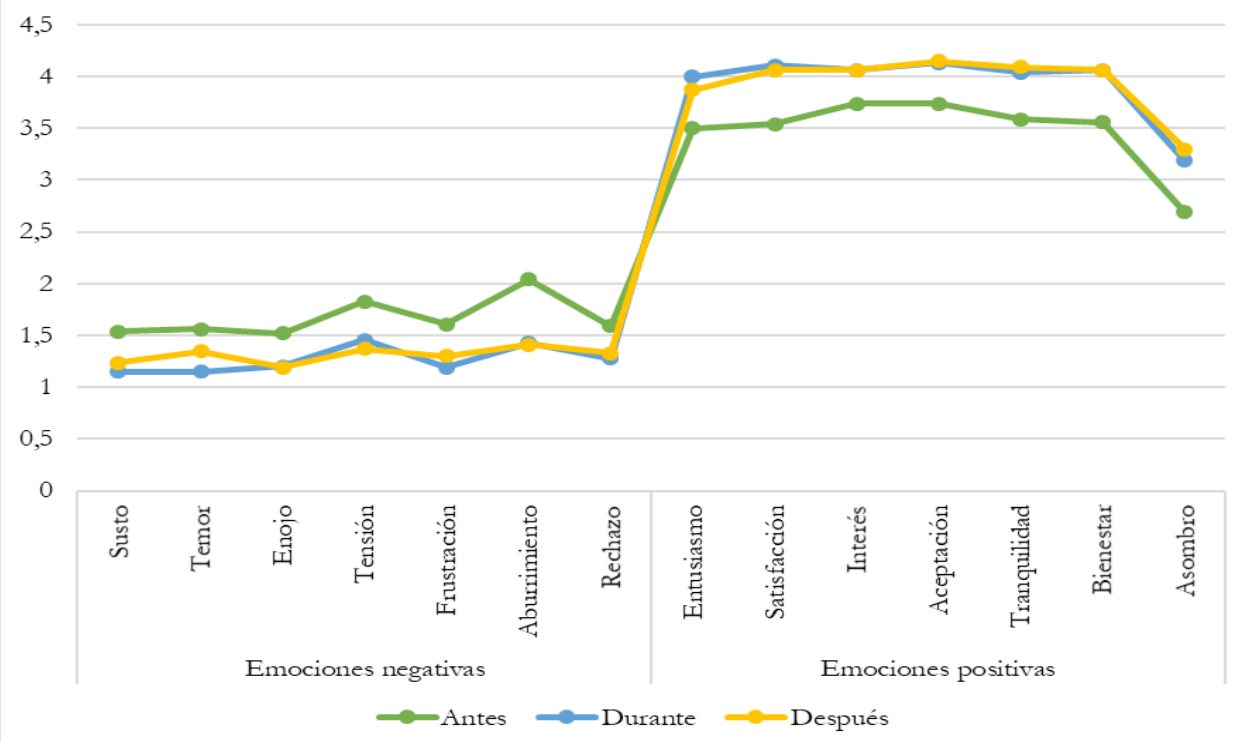

Figura 1. Cambio de emociones de maestros en formación inicial hacia clima de aula.

\section{Análisis inferencial intergrupo}

Para comprobar si existen diferencias significativas o no significativas en intensidad de cada variable y en función del momento, se presentan resultados del análisis intergrupo para tres muestras independientes $(\mathrm{N}=162)$ mediante prueba no paramétrica $\mathrm{H}$ de Kruskal-Wallis. En tabla 3 se incluye estadístico $\mathrm{H}$, significaciones y coeficiente eta cuadrado $\left(\mathrm{E}_{\mathrm{R}}^{2}\right)$ utilizado como estimador mesurado de tamaño del efecto.

Para cada emoción se planteó la hipótesis nula y alternativa:

- $\mathrm{H}_{0}=\mathrm{La}$ mediana en los tres grupos de cada variable de contraste es la misma.

- $\mathrm{H}_{1}=$ La mediana de los tres grupos es diferente.

Tabla 3. Prueba no paramétrica $H$ de Kruskal Wallis para tres muestras no relacionadas en función del momento de la intervención (antes, durante y después) para el clima de aula

\begin{tabular}{cccc} 
para el clima de aula & $\chi^{2}$ & $p$ & $E^{2}{ }_{R}$ \\
\hline Variable & 8,996 &, $011^{*}$ &, 06 \\
Susto & 6,041 &, $049^{*}$ &, 04 \\
Temor & 6,492 &, $039^{*}$ &, 04 \\
Enojo & 8,536 &, $014^{*}$ &, 05 \\
Tensión & 7,832 &, $020^{*}$ &, 05 \\
Frustración & 15,44 &, $000^{*}$ &, 10 \\
Aburrimiento & 5,865 &, 053 &, 04 \\
Rechazo & 7,283 &, $026^{*}$ &, 07 \\
Entusiasmo & 10,88 &, $004^{*}$ &, 03 \\
Satisfacción & 5,152 &, 076 &, 04 \\
Interés & 6,344 &, $042^{*}$ &, 06 \\
Aceptación & 10,44 &, $005^{*}$ &, 04 \\
Tranquilidad & 7,741 &, $021^{*}$ &, $030^{*}$ \\
Bienestar & 7,005 & & \\
Asombro & & & \\
\hline
\end{tabular}

Nota: $* p<, 05$

Se obtuvo que existen diferencias significativas en función de los tres momentos para susto $(\mathrm{p}=, 011)$, temor $(\mathrm{p}=, 049)$, enojo $(\mathrm{p}=, 039)$, tensión $(\mathrm{p}=, 014)$, frustración $(\mathrm{p}=, 020)$, aburrimiento $(\mathrm{p}=, 000)$, entusiasmo $(\mathrm{p}=, 026)$, satisfacción $(\mathrm{p}=, 004)$, aceptación $(\mathrm{p}=, 042)$, tranquilidad $(\mathrm{p}=, 005)$, 
bienestar $(\mathrm{p}=, 021)$ y asombro $(\mathrm{p}=, 030)$. No existen diferencias significativas entre los tres momentos para rechazo $(\mathrm{p}=, 053)$ e interés $(\mathrm{p}=, 076)$. En cuanto a las magnitudes resultantes del coeficiente eta-cuadrado se obtuvo valores bajos en cada variable debido a que los datos provienen de una muestra reducida y el tamaño del efecto se interpreta como pequeño.

Para identificar los pares de momentos en los que sí se establecen diferencias significativas en las variables se realizaron las pruebas post-hoc. En la tabla 4 se presentan los resultados de la prueba no paramétrica $U$ de Mann-Whitney para dos pares de momentos no relacionados.

Tabla 4. Prueba no paramétrica U de Mann-Whitney para las emociones positivas y negativas hacia el clima de aula en función de los momentos de la intervención indagatoria

\begin{tabular}{|c|c|c|c|c|c|c|c|}
\hline Variable & Muestra 1-Muestra 2 & $\chi^{2}$ & $\vec{U}$ & $p$ & $\bar{\beta}$ & $P$ & $T E$ \\
\hline \multirow[t]{3}{*}{ Susto } & Antes-Durante & 18,87 & 1119,5 & ,010* & 10,6 & 89,4 & ,38 \\
\hline & Antes-Después & 12,65 & 1229,0 &, 145 & 31,5 & 68,5 &, 42 \\
\hline & Durante-Después & $-6,213$ & 1571,0 & ,998 & 78,0 & 22,0 &, 54 \\
\hline \multirow{3}{*}{ Temor } & Antes-Durante & 15,55 & 1160,0 & ,045* & 10,3 & 89,7 & ,40 \\
\hline & Antes-Después & 6,222 & 1346,0 & 1,000 & 64,1 & 35,9 &, 46 \\
\hline & Durante-Después & $-10,33$ & 1644,0 &, 387 & 42,0 & 58,0 &, 56 \\
\hline \multirow[t]{3}{*}{ Enojo } & Antes-Durante & 15,70 & 1176,5 & ,049* & 25,9 & 74,1 &, 40 \\
\hline & Antes-Después & 12,74 & 1227,5 &, 155 & 20,7 & 79,3 & 42 \\
\hline & Durante-Después & $-2,963$ & 1512,5 & 1,000 & 93,9 & 6,10 &, 52 \\
\hline \multirow[t]{3}{*}{ Tensión } & Antes-Durante & 19,43 & 1108,5 & ,035* & 33,3 & 66,7 & ,38 \\
\hline & Antes-Después & 19,50 & 1106,5 & ,034* & 13,6 & 86,4 &, 38 \\
\hline & Durante-Después &, 074 & 1457,0 & 1,000 & 85,9 & 14,1 &, 50 \\
\hline \multirow[t]{3}{*}{ Frustración } & Antes-Durante & 19,82 & 1103,0 & ,015* & 8,40 & 91,6 & ,38 \\
\hline & Antes-Después & 10,31 & 1270,5 &, 436 & 32,3 & 67,7 &, 44 \\
\hline & Durante-Después & $-9,509$ & 1631,0 &, 539 & 69,9 & 30,1 &, 56 \\
\hline \multirow[t]{3}{*}{ Aburrimiento } & Antes-Durante & 26,19 & 984,0 & ,003* & 3,20 & 96,8 &, 34 \\
\hline & Antes-Después & 27,69 & 962,0 &, $001 *$ & 3,50 & 96,5 &, 33 \\
\hline & Durante-Después & 1,500 & 1428,5 & 1,000 & 93,4 & 6,60 & 49 \\
\hline \multirow{3}{*}{ Rechazo } & Antes-Durante & - & 1150,0 & - & - & - & ,39 \\
\hline & Antes-Después & - & 1249,0 & - & - & - &, 43 \\
\hline & Durante-Después & - & 1563,5 & - & - & - &, 54 \\
\hline \multirow[t]{3}{*}{ Entusiasmo } & Antes-Durante & $-22,32$ & 1867,0 & ,026* & 9,00 & 91,0 & ,64 \\
\hline & Antes-Después & $-15,92$ & 1737,5 &, 185 & 35,3 & 64,7 &, 60 \\
\hline & Durante-Después & 6,398 & 1350,0 & 1,000 & 79,6 & 20,4 &, 46 \\
\hline \multirow[t]{3}{*}{ Satisfacción } & Antes-Durante & $-26,14$ & 1926,0 & ,006* & 4,10 & 95,9 &, 66 \\
\hline & Antes-Después & $-21,46$ & 1847,0 & ,034* & 9,00 & 91,0 & ,63 \\
\hline & Durante-Después & 4,685 & 1371,0 & 1,000 & 90,5 & 9,50 &, 47 \\
\hline \multirow[t]{3}{*}{ Interés } & Antes-Durante & - & 1749,0 & - & - & - & ,60 \\
\hline & Antes-Después & - & 1756,5 & - & - & - & ,60 \\
\hline & Durante-Después & - & 1462,5 & - & - & - &, 50 \\
\hline \multirow[t]{3}{*}{ Aceptación } & Antes-Durante & $-18,49$ & 1795,0 &, 083 & 20,2 & 79,8 &, 62 \\
\hline & Antes-Después & $-18,17$ & 1781,0 & ,092 & 21,6 & 78,4 & ,61 \\
\hline & Durante-Después &, 315 & 1456,5 & 1,000 & 93,4 & 6,60 &, 50 \\
\hline \multirow[t]{3}{*}{ Tranquilidad } & Antes-Durante & $-22,00$ & 1853,0 & ,026* & 16,7 & 83,3 & ,64 \\
\hline & Antes-Después & $-24,66$ & 1903,0 &, $010 *$ & 9,90 & 90,1 & ,65 \\
\hline & Durante-Después & $-2,66$ & 1505,0 & 1,000 & 91,0 & 9,00 &, 52 \\
\hline \multirow[t]{3}{*}{ Bienestar } & Antes-Durante & $-21,95$ & 1859,0 &, $029 *$ & 12,5 & 87,5 & ,64 \\
\hline & Antes-Después & $-18,49$ & 1785,0 & ,088 & 13,9 & 86,1 & 61 \\
\hline & Durante-Después & 3,463 & 1401,5 & 1,000 & 95,0 & 5,00 &, 48 \\
\hline \multirow[t]{3}{*}{ Asombro } & Antes-Durante & $-19,25$ & 1793,5 & ,084 & 34,5 & 65,5 & ,62 \\
\hline & Antes-Después & $-20,83$ & 1844,0 & 052 & 17,7 & 82,3 & ,63 \\
\hline & Durante-Después & $-1,583$ & 1475,5 & 1,000 & 89,2 & 10,8 &, 51 \\
\hline
\end{tabular}

En el par antes-durante existen diferencias significativas para susto, temor, enojo, tensión, frustración, aburrimiento, entusiasmo, satisfacción, tranquilidad y bienestar. Las probabilidades de cometer errores Tipo II son escasas debido a altas potencias. Se reportan diferencias significativas para el par antes-después en tensión, aburrimiento, satisfacción y tranquilidad. En los pares antes-durante y antesdespués, las diferencias en las emociones negativas se aproximan a un pequeño efecto y las correspondientes a las emociones positivas reportan un tamaño del efecto que se interpreta como mediano, ambos pares con poder superior al 88,0\%, en promedio. Entre el par durantedespués no existen diferencias significativas en ninguna, se reportan tamaños del efecto pequeños que son menores o iguales a ,56, acompañados de muy bajas potencias como para rechazar las hipótesis nulas.

Las emociones que favorecen diferencias significativas en el momento previo son las que 
reportan medianas de mayor intensidad: susto, temor, enojo, tensión, frustración y aburrimiento. Las emociones que favorecen diferencias para el momento durante son entusiasmo, satisfacción, tranquilidad y bienestar. Ninguna emoción favorece diferencias significativas para el momento posterior (ver tabla 4).

\section{Discusión}

Al comienzo de la asignatura, la manera en cómo se presenta el formador, gusto que transmita hacia los contenidos, competencias emocionales que promueva, gestión del espacio e interacciones afectivas que establece podrían condicionar las emociones que maestros en formación vaticinen y experimenten hacia el clima de aula y otras dimensiones. Derivado de la validación del instrumento por prueba piloto se evidenció que, al inicio de la asignatura, los sujetos vaticinaron con mayor intensidad emociones positivas en contraste con emociones negativas. Para ilustrar esto, interés y tensión registraron intensidades medias más altas al comienzo del curso, tendencia que se mantuvo a lo largo de la intervención.

En el momento previo predominan emociones positivas, especialmente interés y aceptación evidenciándose mayor intensidad a diferencia de emociones negativas. Los resultados indican mayor intensidad en todas las emociones negativas a diferencia de los siguientes momentos cuando se aprecia disminución significativa en la mayoría para los pares antes-durante y antesdespués, mas no en durante-después, cuyas diferencias son mínimas. Al inicio, tensión y aburrimiento fueron las emociones negativas que experimentaron principalmente.

En el momento durante se detectó un incremento en todas las emociones positivas, particularmente satisfacción. Una vez más, aceptación registró intensidad más alta; esta implica aprobación y afinidad entre pares y formadora. Todas las emociones negativas experimentaron una caída, en especial aburrimiento y frustración. Al mismo tiempo tensión se mantuvo como la emoción negativa más fuerte que implica excitación.

La formadora contribuyó en establecer un clima emocional adecuado, pues mediante observaciones de clase se apreció que mantuvo una relación cercana con los futuros maestros cuando les preguntaba cómo se sentían y eso estrechaba la relación afectiva. Además, consensuaba un acuerdo didáctico y resaltaba acontecimientos positivos sin castigar acciones erróneas, dudas o temores que surgían; si alguien contestaba de manera equívoca corregía sin dar importancia a cuestiones negativas. También, fomentó trabajo en grupo, momentos de humor y discusiones. Interpretamos que la visita al ecosistema y la actividad de meditación en la playa contribuyó en resultados muy favorables, lo cual se evidencia en caída de todas las emociones negativas e incremento de todas las emociones positivas.

Se detectó que entre el par antes-durante se establecieron diferencias significativas en la mayoría de las emociones negativas excepto en rechazo, entusiasmo, satisfacción, tranquilidad y bienestar. Al finalizar, no todas las emociones positivas incrementaron respecto al momento intermedio, no hubo diferencias significativas entre el par durante-después en ninguna, pero sí en satisfacción y tranquilidad para el par antes-después. Al comparar los momentos previo y final se halló que todas aumentaron, destacándose tranquilidad y bienestar.

Los hallazgos indican que después de la intervención, aceptación, tranquilidad y asombro aumentaron e interés se mostró constante respecto al momento intermedio. Aceptación fue la que más incrementó. Es más, interés junto con bienestar no contribuyó en definición de diferencias significativas entre durante-después. Al comparar los momentos inicial y final, se aprecia que todas las emociones negativas disminuyeron, en particular aburrimiento y tensión, que reportaron diferencias significativas. Al comparar los resultados entre durante y después se detectó que susto, temor, frustración y rechazo se intensificaron levemente, mientras que 
aburrimiento, enojo y tensión disminuyeron sutilmente, hay ausencia de diferencias significativas en todo el conjunto negativo para ese par temporal, lo cual se verifica a partir de bajas potencias y altas probabilidades de cometer errores Tipo II.

Se aprecia que la intervención facilitó el cambio en emociones positivas y negativas frente al clima de aula en los tres momentos. Estos hallazgos son similares a los descritos en estudios previos con futuros docentes (Dávila et al. 2015) en los que mediante intervenciones basadas en metodologías experimentales se evidencia evolución en emociones positivas y disminución en emociones negativas. Si bien, esas evocan emociones hacia contenidos científicos y prácticas de laboratorio, nuestros hallazgos representan una aportación a la Didáctica de las Ciencias Experimentales en España, pues a la fecha no se ha documentado sobre intervenciones de regulación emocional e indagación basada en cuestiones sociocientíficas que enfaticen sobre la dimensión emocional del clima de aula.

A pesar de que se registraron diferencias significativas y potencias altas en la mayoría de las variables según el momento, se obtuvo valores de tamaño del efecto pequeños (emociones negativas) y medianos (emociones positivas) para los pares antes-durante y antes-después correspondientemente. Aunque, el propósito no es la generalización, los resultados son favorables y representan oportunidad para reflexionar sobre estrategias de autorregulación y el rol que desempeñan dentro del modelo de conocimiento profesional de la formadora.

\section{Conclusiones e implicaciones formativas}

El instrumento aporta evidencias óptimas de confiabilidad y validez que permiten describir el cambio de emociones hacia el clima de aula, enriquecer el diseño y aplicación de la intervención. Los resultados permiten aceptar la primera hipótesis pues se establece que las emociones positivas registraron medias más altas en comparación con las emociones negativas a lo largo de toda la intervención.

Al inicio todas las emociones positivas fueron más intensas que las emociones negativas, destacándose satisfacción e interés. En el momento intermedio todas aumentaron, especialmente satisfacción. Al final, se aprecia un incremento en aceptación, tranquilidad y asombro, una merma en entusiasmo y satisfacción y una constante en interés y bienestar. En cuanto a las emociones negativas, mostraron intensidades menores que las emociones positivas en el transcurso de la indagación. Todo el conjunto negativo fue más intenso antes que en los otros momentos. Durante la indagación, todas las emociones negativas disminuyeron y una vez finalizada, incrementó susto, temor, frustración y rechazo y disminuyó enojo, tensión y aburrimiento.

La segunda hipótesis es aceptada parcialmente pues se establecen diferencias significativas en la mayoría en función del momento, excepto en interés y rechazo y no en todos los pares se definen tales diferencias. Para el par temporal antes-durante se hallan diferencias en susto, temor, enojo, tensión, frustración, aburrimiento, entusiasmo, satisfacción, tranquilidad y bienestar. Respecto al par durante-después no existen diferencias significativas en ninguna variable. Acerca del par antesdespués las diferencias son significativas en satisfacción, tranquilidad, tensión y aburrimiento.

Los hallazgos son trasladables a la práctica docente pues admiten reconocer que la indagación facilita en los futuros maestros la mejora en el efecto de sus emociones, en la resolución del problema sociocientífico que requiere implicación cognitiva, emocional y ética. Estos permiten plantear implicaciones formativas:

- La formación inicial debe paliar el efecto de emociones negativas que generaron en futuros maestros un alejamiento hacia las ciencias, debido a modelos didácticos transmisivos en ciclos previos. Intervenciones basadas en problemas sociocientíficos pueden contribuir en 
evolución de emociones positivas y disminución de emociones negativas hacia diversos ámbitos curriculares.

- En la formación docente es necesaria la incorporación de la educación emocional dentro de un modelo de conocimiento profesional que incluya transversalmente el empleo de estrategias de metacognición y autorregulación emocional, de tal forma que las emociones positivas inmersas en el CDC del formador sean transferibles a futuros maestros en un clima de aula idóneo que facilite progresión de madurez emocional y aprendizaje de contenidos científicos y didácticos.

- La educación emocional podría incorporarse en el currículum mediante tratamiento integrado en los contenidos, apoyado de una intervención práctica y reflexiva, pues necesitan adquirir competencias emocionales, bienestar, efectividad y superar obstáculos en el desarrollo profesional. Eso implica que los formadores debemos poseer esas capacidades y enseñarlas mediante el propio modelo didáctico, resaltándolas explícitamente para que las incorporen dentro del repertorio de competencias profesionales. El clima de aula es un requisito previo para conseguir dichas capacidades y empatía en las relaciones interpersonales. Es relevante involucrarles en reflexiones individuales y discusiones grupales acerca de las emociones y consecuencias negativas de una expresión emocional inapropiada.

- La transferencia de los resultados de la investigación en la mejora educativa requiere también formación de maestros en ejercicio, a través de reflexiones sobre y en la práctica para que desarrollen competencias emocionales mientras potencian CDC.

Finalmente, la elección de la escala de niveles no pareció una idea desacertada, pues los coeficientes relacionados con fiabilidad fueron satisfactoriamente aceptables. El estudio forma parte de un trabajo más amplio; el análisis de observaciones de clase, entrevista semiestructurada con la formadora, cuestionarios sobre conocimientos acerca de ecosistema y vídeos del juego de rol, los cuales fueron utilizados como instrumentos de recolección de datos complementarios permitirá atribuir causas a los resultados. Por razones de espacio no se aportan datos al respecto y serán objeto de posteriores publicaciones que lo sustentan.

\section{Agradecimientos}

Este estudio es financiado por la Oficina de Asuntos Internacionales y Cooperación Externa de la Universidad de Costa Rica mediante beca doctoral a Diego Armando Retana Alvarado.

\section{Referencias bibliográficas}

Alderman G. (2011) Classroom Climate. En S. Goldstein, J. A. Naglieri (eds.), Encyclopedia of Child Behavior and Development (pp. 367-370). Springer: New York.

Bellocchi A., Ritchie S. M., Tobin K., King D., Sandhu M., Henderson S. (2014) Emotional climate and high-quality learning experiences in science teacher education. Journal of Research in Science Teaching 51(10), 1301-1325.

Bisquerra R. (2016) 10 ideas clave. Educación emocional. Barcelona: Graó.

Brígido M., Couso D., Gutiérrez C., Mellado V. (2013) The Emotions about Teaching and Learning Science: A Study of Prospective Primary Teachers in Three Spanish Universities. Journal of Baltic Science Education 12(3), 299-311.

Cañal P., Pozuelos F. J., Travé G. (2005) Proyecto Curricular Investigando Nuestro Mundo (6-12). (Vol. 1). Descripción General y Fundamentos. Sevilla: Díada. 
Dávila M. A., Borrachero A. B., Cañada F., Martínez G., Sánchez J. (2015) Evolución de las emociones que experimentan los estudiantes del grado de maestro en educación primaria, en didáctica de la materia y la energía. Revista Eureka sobre Enseñanza y Divulgación de las Ciencias 12(3), 550-564.

Evans I. M., Harvey S. T., Buckley L., Yan E. (2009) Differentiating classroom climate concepts: Academic management, and emotional environments. Kötuitui: New Zealand Journal of Social Science Online 4(2), 131-146.

Fernández-Abascal E. G. (2009) Emociones positivas, psicología positiva y bienestar. En E. G. Fernández-Abascal (Coord.), Emociones positivas (pp. 27-46). Madrid: Ediciones Pirámide.

Frenzel A. C. (2014) Teacher Emotions. En R. Pekrun, L. Linnenbrink-Garcia (eds.), International Handbook of Emotions in Education (pp. 494-519). New York: Routledge, Taylor and Francis.

Gess-Newsome J. (2015) A model of teacher professional knowledge and skill including PCK: Results of the thinking from the PCK Summit. En A. Berry, P. Friedrichsen, J. Loughran (eds.), Re-examining Pedagogical Content Knowledge in Science Education (pp. 28-42). New York: Routledge.

Hagenauer G., Hascher T., Volet S. (2015) Teacher emotions in the classroom: associations with students' engagement, classroom discipline and the interpersonal teacher-student relationship. European Journal of Psychology of Education 30(4), 385-403.

López-González L., Oriol X. (2016) The relationship between emotional competence, classroom climate and school achievement in high school students. Culture and Education 28(1), 130-156.

Matas A. (2018) Diseño del formato de escalas tipo Likert: un estado de la cuestión. Revista Electrónica de Investigación Educativa, 20(1), 38-47.

Mellado V., Borrachero A. B, Brígido M., Melo L. V., Dávila M. A., Cañada F., Conde M. C., Costillo E., Cubero J., Esteban R., Martínez G., Ruiz C., Sánchez J., Garritz A., Mellado L., Vázquez-Bernal B., Jiménez-Pérez R., Bermejo M. L. (2014) Las emociones en la enseñanza de las ciencias. Enseñanza de las Ciencias 32(3), 11-36.

Melo L. V., Cañada F., Mellado V. (2017) Exploring the emotions in Pedagogical Content Knowledge about the electric field. International Journal of Science Education 39(8), 10251044.

Mora F. (2016) Neuroeducación. Solo se puede aprender aquello que se ama. Madrid: Alianza Editorial.

Retana-Alvarado D. A., de las Heras-Pérez M. Á., Jiménez-Pérez R., Vázquez-Bernal B. (2017) Emociones de maestros en formación inicial sobre la Didáctica de las Ciencias antes de una intervención indagatoria. Enseñanza de las Ciencias no extraordinario, 5415-5421.

Ritchie S. M., Tobin K., Sandhu M., Sandhu S., Henderson S., Roth W. (2013) Emotional arousal of beginning physics teachers during extended experimental investigations. Journal of Research in Science Teaching 50(2), 137-161.

Shapiro S. (2010) Revisiting the teachers' lounge: Reflections on emotional experience and teacher identity. Teaching and Teacher Education 26, 616-621.

Shulman L. S. (1986) Those who understand: knowledge growth in teaching. Educational Research 57(1), 1-22. 
Sutton R. E., Harper E. (2009) Teachers' Emotion Regulation. En L. J. Saha, A. G. Dworkin (eds.), International Handbook of Research on Teachers and Teaching (pp. 389-402). Springer: New York.

Tomas L., Rigano D., Ritchie S. (2016) Students' Regulation of Their Emotions in a Science Classroom. Journal of Research in Science Teaching 53(2), 234-260.

Uitto M., Jokikokko K., Estola E. (2015) Virtual special issue on teachers and emotions in Teaching and teacher education (TATE) in 1985-2014. Teaching and Teacher Education 50, 124135.

Vázquez-Bernal B., Mellado V., Jiménez-Pérez R., Taboada M. C. (2012) The process of change in a science teacher's professional development: A case study based on the types of problems in the classroom. Science Education 96(2), 337-363.

Zeidler D. L. (2014) Socioscientific Issues as a Curriculum Emphasis: Theory, Research, and Practice. En N. G. Lederman, S. Abell (eds.), Handbook of Research on Science Education (Vol. 2) (pp. 697-726). New York: Routledge. 
Anexo 1.

Cuestionario: Emociones de estudiantes de1 Grado en Maestro de Educación Primaria.

Este cuestionario forma parte de una investigación cuyo objetivo es mejorar el proceso de enseñanza y aprendizaje. Completa el cuestionario con bolígrafo de tinta azul o negra y responde con la mayor sinceridad. Asegúrate que has respondido todas las preguntas. Muchas gracias por tu colaboración.

I Parte. Información general del estudiante. Responde los ítems marcando una equis $(\mathrm{X})$ dentro del paréntesis correspondiente $o$ indica la respuesta en el espacio que se te proporciona.

1. Sexo: ( ) Hombre ( ) Mujer

2. Edad:

3. Grupo en el cual estás matriculado(a):

4. Procedencia:

5. Acceso a la universidad:

() Bachillerato (pasa al ítem 6) () Formación Profesional (pasa al ítem 7) () Prueba $>25$ años

6. Modalidad de Bachillerato:

() Artes ( ) Ciencias de la Naturaleza y Salud ( ) Tecnología ( ) Humanidades ( ) Ciencias Sociales 7. Modalidad de Formación Profesional:

II Parte. Emociones hacia el clima de aula.

Lee la lista de emociones que se exponen a continuación y consulta el glosario de emociones. Luego marca el número que mejor describa la emoción que sientes frente al aspecto resaltado. Asegúrate de marcar solamente un número para cada emoción. Los números están organizados según el grado de acuerdo de las emociones que manifiestas.

1. ¿Qué emociones sientes en relación con el clima de aula (interacción socioafectiva entre estudiantes y profesor, normas de clase, espacio y situaciones de aprendizaje)?

\begin{tabular}{|c|c|c|c|c|c|}
\hline Emociones & $\begin{array}{c}\text { Totalmente en } \\
\text { desacuerdo }\end{array}$ & $\begin{array}{c}\text { En } \\
\text { desacuerdo }\end{array}$ & $\begin{array}{c}\text { Ni de acuerdo } \\
\text { ni en } \\
\text { desacuerdo }\end{array}$ & $\begin{array}{c}\text { De } \\
\text { acuerdo }\end{array}$ & $\begin{array}{l}\text { Totalmente } \\
\text { de acuerdo }\end{array}$ \\
\hline Susto & (1) & (2) & (3) & (4) & (5) \\
\hline Entusiasmo & (1) & (2) & (3) & (4) & (5) \\
\hline Temor & (1) & (2) & (3) & (4) & (5) \\
\hline Satisfacción & (1) & (2) & (3) & (4) & (5) \\
\hline Enojo & (1) & (2) & (3) & (4) & (5) \\
\hline Interés & (1) & (2) & (3) & (4) & (5) \\
\hline Tensión & (1) & (2) & (3) & (4) & (5) \\
\hline Aceptación & (1) & (2) & (3) & (4) & (5) \\
\hline Frustración & (1) & (2) & (3) & (4) & (5) \\
\hline Tranquilidad & (1) & (2) & (3) & (4) & (5) \\
\hline Aburrimiento & (1) & (2) & (3) & (4) & (5) \\
\hline Bienestar & (1) & (2) & (3) & (4) & (5) \\
\hline Rechazo & (1) & (2) & (3) & (4) & (5) \\
\hline Asombro & (1) & (2) & (3) & (4) & (5) \\
\hline
\end{tabular}

Glosario de emociones

\begin{tabular}{|c|c|}
\hline Emoción & Definición conceptual \\
\hline Susto & Es una impresión repentina causada por miedo. \\
\hline Entusiasmo & $\begin{array}{l}\text { Surge en respuesta a conseguir una meta u objetivo. La persona llega a poner } \\
\text { en su logro mucho empeño. }\end{array}$ \\
\hline Temor & $\begin{array}{l}\text { Surge de la interpretación acerca de si la situación que enfrenta es peligrosa y } \\
\text { constituye una amenaza para su bienestar. }\end{array}$ \\
\hline Satisfacción & Se manifiesta cuando se ha alcanzado un deseo o cubierto una necesidad. \\
\hline Enojo & $\begin{array}{l}\text { Proviene de la restricción o interpretación de que alguna barrera u obstáculo } \\
\text { ha interferido con los propios planes, metas o bienestar. }\end{array}$ \\
\hline Interés & $\begin{array}{l}\text { Emoción que crea el deseo de explorar, investigar, buscar, manipular, extraer } \\
\text { información de los objetos que nos rodean. }\end{array}$ \\
\hline Tensión & Se caracteriza por la excitación en relación con determinadas situaciones. \\
\hline Aceptación & $\begin{array}{l}\text { Implica aprobación, reconocimiento y afinidad (atracción o compatibilidad en- } \\
\text { tre dos o más personas o entre una persona y algo). }\end{array}$ \\
\hline Frustración & $\begin{array}{l}\text { Surge de experiencias de fracaso, es decir, se manifiesta cuando el progreso } \\
\text { hacia un objetivo deseado se ve interrumpido. }\end{array}$ \\
\hline Tranquilidad & Es una emoción que se caracteriza por la presencia de serenidad en la persona. \\
\hline Aburrimiento & $\begin{array}{c}\text { Es una emoción que se genera por falta de interés o cuando no logras impli- } \\
\text { carte en una actividad satisfactoria. }\end{array}$ \\
\hline Bienestar & Es una emoción que responde al propósito consciente de ser feliz. \\
\hline Rechazo & Emoción que implica alejarse o resistirse a algo. \\
\hline Asombro & Reacción causada por algo imprevisto, novedoso o extraño. \\
\hline
\end{tabular}

